\title{
Differential gene expression of the toll-like receptor-4 cascade and neutrophil function in early- and mid-lactating dairy cows
}

\author{
M. G. H. Stevens, ${ }^{*}$ L. J. Peelman,† B. De Spiegeleer,‡ A. Pezeshki, ${ }^{\star}$ G. R. Van De Walle, ${ }^{\star}$ L. Duchateau, ${ }^{\star}$ \\ and C. Burvenich*1 \\ *Department of Comparative Physiology and Biometrics, Faculty of Veterinary Medicine, and \\ †Department of Nutrition, Genetics and Ethology, Faculty of Veterinary Medicine, Ghent University, B-9820 Merelbeke, Belgium \\ ‡Drug Quality and Registration (DruQuaR) group, Faculty of Pharmaceutical Sciences, Ghent University, B-9000 Ghent, Belgium
}

\section{ABSTRACT}

During early lactation, neutrophils display several reduced immune functions. Particularly, a delayed recruitment of neutrophils into the infected udder seems to be one of the underlying events involved in the severity of postpartum Escherichia coli intramammary infections. The purpose of this study was to analyze the effect of in vitro chemotaxis and diapedesis on the expression of toll-like receptor-4 (TLR4)-related genes in bovine blood neutrophils isolated from 10 early-lactating (EL) and 10 mid-lactating (ML) cows. Functional characterization of the neutrophil population was performed by measuring phagocytosis and production of reactive oxygen species (chemiluminescence). Messenger RNA was extracted from neutrophils, and the expression of TLR4 and associated genes in EL and ML cows was analyzed by reverse-transcription quantitative PCR. To study the effect of chemotaxis and diapedesis on the expression of genes of the TLR4 cascade, neutrophils were stimulated to (trans)migrate in response to $\mathrm{C} 5 \mathrm{a}$ using in vitro models. Our salient findings were that both neutrophil migration in vitro and lactation stage induced significant changes in the expression of several genes of the TLR4 signaling cascade. Before migration, expression of TRAF6, ATF3, RELA, IL8, and C5aR were lower in EL than in ML cows. Diapedesis and chemotaxis induced an increase in expression of TLR4, ATF3, and IL8 in both EL and ML cows. Diapedesis resulted in a downregulation of Syk, a TLR4-associated gene, in ML cows. This study shows that the perturbations in neutrophil functions during EL are accompanied by modulation of TLR 4 pathway genes. These data can contribute to the understanding of the mechanisms explaining the relationship between stage of lactation and risk of severe $E$. coli mastitis.

Received June 25, 2010.

Accepted October 24, 2010.

${ }^{1}$ Corresponding author: christian.burvenich@ugent.be
Key words: diapedesis, chemotaxis, toll-like receptor-4 (TLR4), neutrophil

\section{INTRODUCTION}

The innate immune system is the most important defense mechanism in the bovine mammary gland. When pathogens develop in the udder cisterns in sufficient numbers, an inflammatory response is activated. Inflammation starts with the migration of neutrophils toward the site of infection and involves a 2 -step process of diapedesis and chemotaxis. Neutrophil migration is one of the key phenomena during the inflammatory process and dysfunction can lead to chronic and even fatal infections such as mastitis in dairy cattle (Burvenich et al., 1994).

Mastitis, an inflammation of the mammary gland, has important effects on both animal health and economic loss in the dairy industry. The severity of symptoms during clinical Escherichia coli mastitis, reflecting the involvement of sepsis, is mainly determined by host factors (Burvenich et al., 2003). In particular, lactation stage and parity of the cow have important effects on mastitis severity. Around parturition, cows are more susceptible to the development of severe mastitis, which is partly explained by the fact that these cows have poor neutrophil recruitment to the udder (Vangroenweghe et al., 2005). Cows in mid lactation show a rapid influx of neutrophils into the infected mammary gland. This massive influx of neutrophils results in a mild to moderate inflammatory response and fast curing of the infection. Thus, neutrophil migration to the site of infection seems a very important factor for the innate immune response.

The involvement of the toll-like receptor (TLR)-4 pathway in the development of bovine $E$. coli mastitis has been hypothesized by De Schepper et al. (2008). When gram-negative bacteria such as E. coli enter the mammary gland, they shed LPS. Neutrophils recognize this pathogen-associated molecular pattern (PAMP) through TLR4 (Beutler et al., 2001). 
However, TLR4 does not recognize LPS by itself. It requires the help of CD14 and myeloid differentiation protein 2 (MD2; Fitzgerald et al., 2004). The binding of LPS to the TLR4/CD14/MD2 receptor complex activates downstream signaling, which can occur via an adaptor protein myeloid differentiation factor 88 (MyD88)-dependent or a MyD88-independent pathway [also known as the TIR-containing adaptor molecule-1 (TICAM-1)-dependent pathway; Akira and Hoshino, 2003). Signaling via MyD88 subsequently results in the activation of the tumor necrosis factor- $\alpha$ receptor-associated factor-6 (TRAF-6), which forms a complex with mitogen-activated protein kinase kinase kinase 7 (MAP3K7). This leads to the activation of a kinase complex that consists of $\alpha(\mathbf{I K K} \mathbf{\alpha})$ and $\beta$ subunits (Akira and Hoshino, 2003). Eventually, the nuclear factor- $\kappa \mathrm{B}(\mathbf{N F}-\boldsymbol{\kappa} \mathbf{B})$ complex is translocated to the nucleus and the transcription of proinflammatory genes (e.g., IL8) is initiated. The NF- $\kappa \mathrm{B}$ complex consists of several subunits that are members of a family of structurally related proteins. Five different Rel proteins (also called Rel/NF-kB proteins) have been identified so far: p50, p52, p65 (also called RELA), RelB, and c-Rel (Ghosh et al., 1998).

Toll-like receptor-4 signaling is strictly regulated by endogenous inhibitory molecules that act at different levels of the signaling cascade. The E3 ubiquitin-protein ligase (Triad3A) interacts with the toll/IL-1 receptor domain of TLR and promotes their proteolytic degradation (Chuang and Ulevitch, 2004). Phosphatidylinositol 3-kinase (PI3K) regulates TLR4 signaling at the plasma membrane, and PI3K activation leads to a depletion of $\mathrm{PIP}_{2}$ stores at the membrane, resulting in the dissociation of TIRAP, which forms a bridge between TLR4 and MyD88 and thereby, diminishes TLR4-dependent signaling (Laird et al., 2009). In macrophages it was observed that the activating transcription factor 3 (ATF-3) inhibited IL6 and IL12b transcription by altering chromatin structure, thereby restricting access to transcription factors. It was also shown that ATF3 itself is induced by LPS and it was concluded that ATF3 regulates a TLR4-dependent inflammatory response in a negative- feedback loop (Gilchrist et al., 2006).

Spleen tyrosine kinase (Syk) is a nonreceptor tyrosine kinase most abundant in cells of the hematopoietic lineages (Sada et al., 2001); Syk is involved in a variety of biological functions such as cell adhesion, innate pathogen recognition, platelet activation, vascular development, and osteoclast maturation (see review by Mócsai et al., 2010). It is usually not shown in the traditional presentation of the TLR 4 cascade but it has been reported that Syk is associated with the TLR4 receptor (Arndt et al., 2004; Chaudhary et al., 2007).
We hypothesized that the diapedesis process itself might alter the TLR4 signaling pathway in such a way that neutrophils from cows in early lactation are not able to induce an adequate TLR4-mediated innate response. To our knowledge nothing is known yet about the effect of neutrophil migration on TLR4 signaling in neutrophils. Therefore, this preliminary study was done to analyze the effect of diapedesis and chemotaxis on the expression of members of the TLR4 signaling cascade in neutrophils from cows in early and mid lactation.

\section{MATERIALS AND METHODS}

\section{Animals}

Ten Holstein-Friesian cows 3 d postpartum (early lactation, EL) and 10 cows 149 to $151 \mathrm{~d}$ postpartum (mid lactation, ML) were used. Cows were housed at the dairy farm (Biocenter Agri-Vet, Melle, Belgium) of Ghent University, and all animals were clinically healthy. Milk production ranged between 6,461 and $14,147 \mathrm{~kg} /$ yr with percentages of fat and protein in milk of 4.23 and 3.43 , respectively. Somatic cell count was $<200,000$ cells $/ \mathrm{mL}$ for all cows. Cows were milked with a milk robot (DeLaval VMS, Drongen, Belgium) at an average voluntary milking frequency of $2.7 / \mathrm{d}$. Cow data (e.g., last milking time, milk yield, food quantity) were measured through a transmitter in the collar of each cow and uploaded into a computer. All cows had access to a TMR during the day. Approval from the local ethical committee (Ghent University) was obtained before commencement of the study (EC2008/108).

\section{Study Design}

Figure 1 shows an overview of the experimental study design. First, neutrophils from EL and ML cows were isolated and functionally characterized by measuring phagocytosis, oxidative burst, diapedesis (DIA), and chemotaxis (CTX) capacity, as described below. Based on these assays, neutrophils from EL and ML cows were characterized as immune-compromised and immunecompetent, respectively. Then, RNA was isolated from unstimulated neutrophils in both groups and the expression levels of important genes of the TLR4 pathway were determined by reverse-transcription quantitative PCR (RT-qPCR). To analyze the effect of DIA on the TLR4 pathway, neutrophils were allowed to perform DIA through an epithelial cell layer in response to C5a. After DIA, neutrophils that performed DIA were quantified and RNA was isolated. The expression of the selected genes was measured by RT-qPCR and 
compared with that of unstimulated neutrophils before migration. The effect of CTX on the TLR4 pathway in neutrophils from EL and ML cows was also studied. Therefore, neutrophils were allowed to migrate through a porous membrane in response to a C5a gradient. After migration, cells were quantified and RNA was isolated for expression analysis. Because C5a was used as a chemoattractant in the DIA and CTX assays, the expression of the $\mathrm{C} 5 \mathrm{a}$ receptor $(\mathbf{C 5 a R})$ was analyzed (Figure 1).

\section{Blood Sampling and Neutrophil Purification}

Blood samples for neutrophil function determination were collected aseptically via the coccygeal vein from EL and ML cows as described by Sears et al. (1978). Fiftymilliliter blood samples from each cow were collected in Vacutainer tubes (Becton Dickinson, Erembodegem, Belgium) containing $150 \mu \mathrm{L}$ of EDTA as anticoagulant. Blood was processed within $0.5 \mathrm{~h}$ of collection. Isolation of neutrophils was performed according to Siemens et

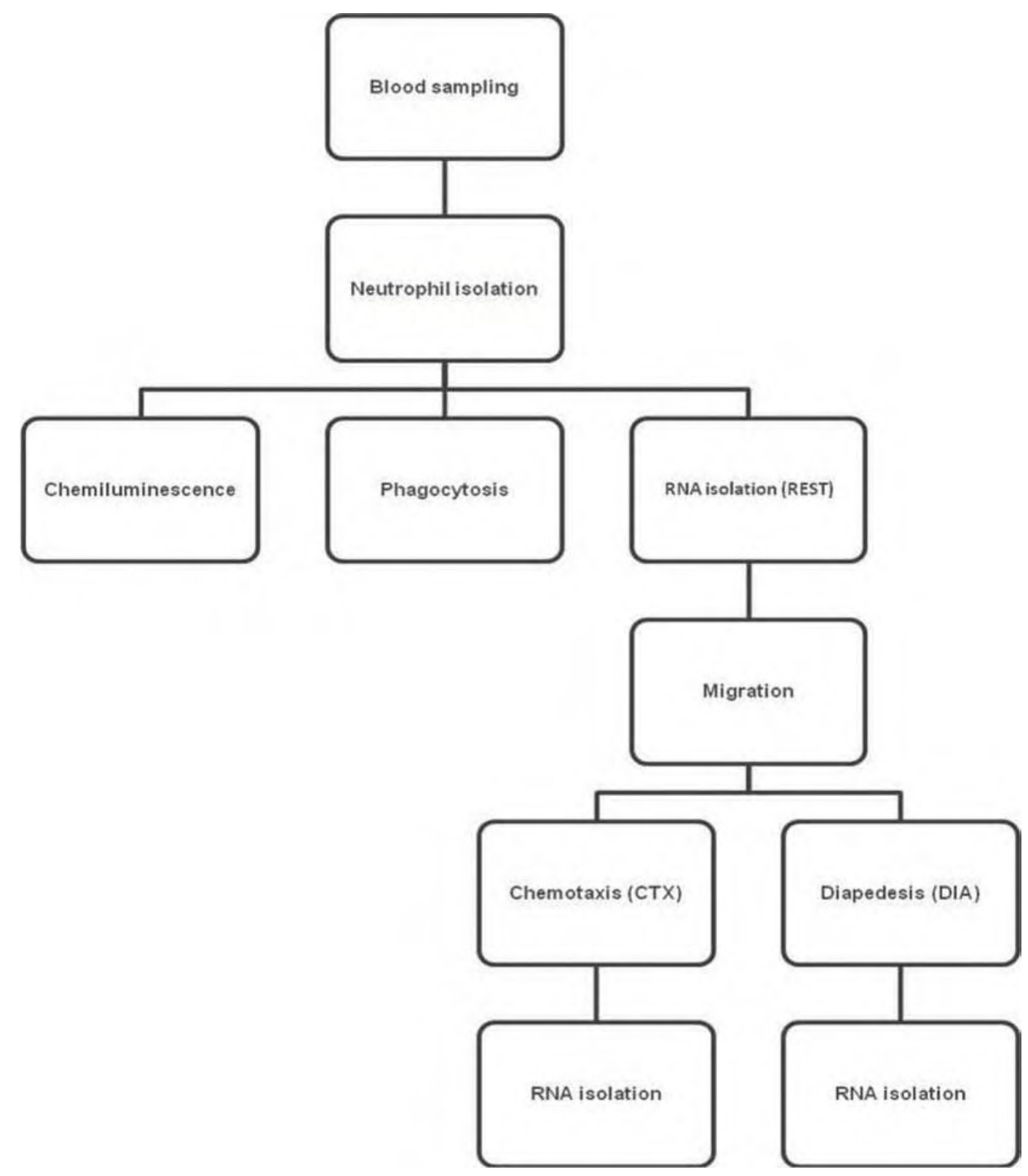

Figure 1. Schematic overview of the study design. After isolation from early- (EL) and mid- (ML) lactating cows, blood polymorphonuclear neutrophils were characterized by chemiluminescence and phagocytosis capacity. mRNA expressions from genes in the TLR4 pathway and associated genes were measured by reverse transcription-quantitative PCR before and after chemotaxis (CTX) or diapedesis (DIA) through in vitro systems. REST = unstimulated cells. 
al. (2007). Briefly, red blood cells were lysed by adding $50 \mathrm{~mL}$ of RNase-free purified water. Five milliliters of $10 \% \mathrm{NaCl}$ was added and the sample was centrifuged at $585 \times g$ for $10 \mathrm{~min}$. Supernatant was discarded and the pellet was resuspended in Hanks' buffered salt solution (HBSS). Ten milliliters of the leukocyte suspension was layered on top of the Histopaque 1077/1119 gradient (Sigma-Aldrich, Bornem, Belgium) and centrifuged at $440 \times g$ for $25 \mathrm{~min}$. After removal of the supernatant, neutrophils were washed by adding $50 \mathrm{~mL}$ of HBSS and centrifuged at $585 \times g$ for $10 \mathrm{~min}$. All centrifugation steps occurred at room temperature $\left(\mathbf{R T}, 20^{\circ} \mathrm{C}\right)$. The final pellet was resuspended in $1 \mathrm{~mL}$ of PBS and stored on ice until used.

Cell viability was assessed with the Trypan blue exclusion test. Therefore, $10 \mu \mathrm{L}$ of the neutrophil suspension was transferred to a microcentrifuge tube and $590 \mu \mathrm{L}$ of a $0.4 \%$ Trypan blue solution (Sigma-Aldrich) was added. Cell counting was done in triplicate using a Bürker chamber. To determine purity, neutrophils were differentiated in smears and stained with Sangodiff G (Merck, Darmstadt, Germany). Slides were analyzed by bright-field microscopy, with a $100 \times$ objective and immersion oil (Axioskop 2 MOT, Carl Zeiss N.V., Zaventem, Belgium).

\section{Neutrophil Function Assays}

Reagents. Ham's F12, RPMI-1640, andNCTC 135 media, and fetal bovine serum, lactose, lactalbumin hydrolysate, reduced L-glutathione, L-ascorbic acid, hydrocortisone, bovine insulin, antibiotic-antimycotic solution, $0.1 \%$ calfskin collagen (type I) solution, HBSS $\left(\mathrm{Ca}^{2+}\right.$ and $\left.\mathrm{Mg}^{2+}\right)$, phorbol 12-myristate 13-acetate (PMA), luminol, and endotoxin-free and ultrafiltered water were purchased from Sigma-Aldrich. Bovine serum albumin was purchased from Roche Diagnostics (Vilvoorde, Belgium).

Phagocytosis Assay. The Vybrant Phagocytosis Assay kit (Invitrogen, Merelbeke, Belgium)was used for nonopsonized phagocytosis of $E$. coli according to Siemens et al. (2007) with some modifications for the fluorescence microscopy analysis. In this study, 0.5 $\mathrm{mL}$ of RPMI-1640 was added to $1 \mathrm{~mL}$ of fluorescein isothiocyanate (FITC) to label the chemically killed E. coli (K-12 strain). Prior to use, the E. coli suspension was incubated for $30 \mathrm{~min}$ in a shaking water bath $\left(37^{\circ} \mathrm{C}\right)$. One million neutrophils were suspended in 1 $\mathrm{mL}$ of RPMI-1640 $\left(37^{\circ} \mathrm{C}\right)$. One hundred fifty microliters of RPMI-1640, $100 \mu \mathrm{L}$ of neutrophils, and $10 \mu \mathrm{L}$ of $E$. coli suspension were aliquoted in $1.5-\mathrm{mL}$ polypropylene microcentrifuge tubes. The reaction was done in triplicate. The final neutrophil: $E$. coli ratio was 1:20. Neutrophils and E. coli were incubated for $20 \mathrm{~min}$ at $37^{\circ} \mathrm{C}$ and $5 \% \mathrm{CO}_{2}$. The reaction was stopped by adding $4 \%$ cold paraformaldehyde. Trypan blue was used to quench extracellular fluorescence and to distinguish attached from ingested bacteria. Phagocytosis was examined with a fluorescence microscopy and phase contrast. For fluorescence, a $100 \times$ immersion oil lens and a FITC filter set (excitation: BP485/20; emission: BP515-565) was used. One hundred neutrophils were counted per sample and the following parameters were calculated: mean number of particles per neutrophil, the percentage of neutrophils that performed phagocytosis, and the phagocytotic index, which was calculated using the following formula:

$$
\left(\frac{N_{b a c}}{N_{\text {neutrophils }}}\right) \times \% N P,
$$

where $N_{b a c}=$ total number of bacteria; $N_{\text {neutrophils }}=$ total number of neutrophils; and $\% N P=$ percentage of neutrophils containing at least 1 bacterium.

Chemiluminescence Assay. Neutrophils were stimulated with or without PMA and luminol was used as luminescent agent as described by Mehrzad et al. (2001). Briefly, 400,000 neutrophils were suspended in Dulbecco's PBS and transferred to a 96-well plate. Luminol $(0.30 \mathrm{~mol} / \mathrm{L})$ and PMA (200 $\mathrm{ng} / \mathrm{mL})$ were added, and PMA was replaced by Dulbecco's PBS for CL measurements from unstimulated neutrophils. All reactions were performed in duplicate. Results were included if $\mathrm{CV}$ was $<10 \%$. Chemiluminescence was measured with a luminometer (type LB96P, EG\&G Berthold, Bad Wildbad, Germany). The area under the curve (AUC) was calculated over a period of $30 \mathrm{~min}$ and expressed as relative light units (RLU). The CL response was corrected for the actual number of viable neutrophils in each sample (Heyneman et al., 1990; Hoeben et al., 2000).

DIA Assay. Bovine mammary epithelial cells (BME-UV) were cultured in $75-\mathrm{cm}^{2}$ tissue culture treated, vented flasks at $37^{\circ} \mathrm{C}$ in $5 \% \mathrm{CO}_{2}$. Cells were subcultured once every 3 to $5 \mathrm{~d}$ when flasks were approximately $80 \%$ confluent. Then, $3 \times 10^{5} \mathrm{BME}-\mathrm{UV}$ cells were added to Millicell-PCF inserts (12-mm diameter, 8.0- $\mu \mathrm{m}$ pore size; Millipore, Bedford, MA) coated with $0.10 \%$ calfskin collagen type I. Models were cultured as described by Smits et al. (1996). The confluence of the epithelial monolayers was determined by epithelial resistance $\left(\Omega \cdot \mathrm{cm}^{2}\right)$ as follows: collagen-coated inserts without cells were used to determine baseline resistance and monolayer resistance was corrected using the following formula: $\left(R_{\text {measured }}-R_{\text {baseline }}\right) \times 0.60 \mathrm{~cm}^{2}(R$, $\Omega)$. In general, monolayers had an average resistance 
of $96.65 \pm 4.11 \Omega \cdot \mathrm{cm}^{2}$ at $\mathrm{d} 14$. Before use, cultures were washed with HBSS (containing $\mathrm{Ca}^{2+}$ and $\mathrm{Mg}^{2+}$ ) to remove the culture medium and incubated for $30 \mathrm{~min}$ with $\mathrm{HBSS}$ at $37^{\circ} \mathrm{C}$ and $5 \% \mathrm{CO}_{2}$. Next, the cultures were transferred to a 24 -well tissue culture dish containing $600 \mu \mathrm{L}$ of HBSS with $0.1 \%$ BSA and $50 \mathrm{ng} / \mathrm{mL}$ of bovine purified complement factor C5a (courtesy of P. Rainard, INRA UR1282 Laboratoire d'Infectiologie Animale et Santé Publique, Nouzilly, France). Then, 20 $\times 10^{6}$ neutrophils were suspended in preheated HBSS with $\mathrm{Ca}^{2+}$ and $\mathrm{Mg}^{2+}\left(37^{\circ} \mathrm{C}\right)$ and added at the basal side of the epithelial cell monolayer. Neutrophils were allowed to perform diapedesis during $90 \mathrm{~min}$ at $37^{\circ} \mathrm{C}$ and $5 \% \mathrm{CO}_{2}$. Transmigrated neutrophils were counted by using a hemocytometer.

CTX Assay. Millicell-PCF inserts (12-mm diameter, 8.0- $\mu \mathrm{m}$ pore size; Millipore) were incubated for 30 min with HBSS at $37^{\circ} \mathrm{C}$ and $5 \% \mathrm{CO}_{2}$ and transferred to a 24 -well tissue culture dish containing $600 \mu \mathrm{L}$ of HBSS with $0.1 \%$ BSA and $50 \mathrm{ng} / \mathrm{mL}$ of bovine purified complement factor C5a. Then, $20 \times 10^{6}$ neutrophils were suspended in preheated HBSS with $\mathrm{Ca}^{2+}$ and $\mathrm{Mg}^{2+}\left(37^{\circ} \mathrm{C}\right)$ and added at the apical chamber of the filter. Neutrophils were allowed to migrate through the filter for $90 \mathrm{~min}$ at $37^{\circ} \mathrm{C}$ and $5 \% \mathrm{CO}_{2}$, and migrated neutrophils were counted with a hemocytometer.

\section{TLR4 Signaling Cascade Before and After CTX and DIA}

The expression of genes of the TLR4 pathway was determined in neutrophils of EL and ML cows with emphasis on the effect of diapedesis and chemotaxis.

RNA Isolation and cDNA Synthesis. Total RNA was extracted from the neutrophils using Total RNA Isolation Reagent (ABgene, Epsom, Surrey, UK), according to the manufacturer's protocol. The obtained RNA was dissolved in $15 \mu \mathrm{L}$ of preheated MilliQ water $\left(65^{\circ} \mathrm{C}\right)$ buffered with Tris-HCl (pH 8.0) (VWR International, Leuven, Belgium). The RNA concentration of the samples was measured with the Nanodrop ND-1000 spectrophotometer (Isogen Life Science, Sint-PietersLeeuw, Belgium), and was between 0.70 and $63.42 \mathrm{ng} /$ $\mu \mathrm{L}$. The quality of RNA was assessed by evaluation of the $28 \mathrm{~S}$ and the $18 \mathrm{~S}$ ribosomal bands on a $0.8 \%$ agarose gel. To remove genomic DNA, digestion was carried out by adding $4 \mu \mathrm{L}$ of RQ1 RNase-free DNase (Promega, Leiden, the Netherlands) and $2 \mu \mathrm{L}$ of RQ1 DNase Reaction Buffer (Promega) to $14 \mu \mathrm{L}$ RNA, followed by spin-column purification (Microcon YM-100, Millipore, Brussels, Belgium). To assure that DNase treatment was successful, a minus RT control PCR was performed on the iCycler (Bio-Rad) in a total volume of $15 \mu \mathrm{L}$ containing $2 \times$ KAPA SYBR FAST qPCR
Master Mix Bio-Rad iCycler (KAPA Biosystems, Woburn, MA), GAPDH primers (Table 1) and $2.5 \mu \mathrm{L}$ of template (RNA, genomic DNA as positive control, and water as negative control). The $\mathrm{qPCR}$ reaction consisted of a 3 -min enzyme activation step at $95^{\circ} \mathrm{C}$, followed by 40 amplification cycles (denaturation for $3 \mathrm{~s}$ at $95^{\circ} \mathrm{C}$ and annealing/elongation/SYBR detection for $30 \mathrm{~s}$ at $60^{\circ} \mathrm{C}$ ) and a melt curve analysis (from 75 to $90^{\circ} \mathrm{C}$ with $0.3^{\circ} \mathrm{C}$ increments of $10 \mathrm{~s}$ ). The amplification products were additionally analyzed on a $2 \%$ agarose gel (results not shown). The DNase-treated RNA was used for generating single-stranded cDNA by means of the Improm II reverse transcription system (Promega) using Random and Oligo dT primers (each $0.80 \mu \mathrm{L}$ per reaction). The conversion was confirmed by a PCR with GADPH primers (giving an amplicon of different lengths on gDNA and cDNA) on $10 \times$ diluted cDNA. All PCR assays included a positive control and a notemplate control.

Reference Gene Selection and Primer Design. Reference genes used for normalization of the data are shown in Table 1 (De Ketelaere et al., 2006). To assess the stability of 6 selected candidate reference genes, their cycle threshold $(\mathbf{C t})$ values were measured on 5 $\times 10^{6}$ neutrophils $(10 \times$ diluted $)$. The reactions were performed in duplicate in 2 independent runs. A correction for technical inter-run variation was carried out as described by Vandesompele et al. (2002). After calibration, the average $\mathrm{Ct}$ value of each sample was converted to raw data (not normalized quantitative data) using the measured gene-specific PCR efficiencies, and the stability of the different reference genes was assessed with the geNorm software (Vandesompele et al., 2002). After identification of the most stable reference genes, $\mathrm{Ct}$ values and raw data for these genes were measured and calculated on all EL and ML samples, as described previously. The primers for the TLR4 receptor and associated target genes were based on the mRNA sequences of the bovine genes (http://www.ncbi.nlm.nih. gov), and the primers were designed using the Primer3 software (http://www.sk.embnet.org/cgi-bin/primer3_ www.cgi). The optimal annealing temperature was set at $60^{\circ} \mathrm{C}$, the optimal $\mathrm{GC}$ content at $50 \%$, and the maximal self-complementarity was kept as low as possible. The presence of secondary structures in the amplicon was checked using 'mfold' (http://www.bioinfo/rpi. edu/applications/mfold/dna), and the specificity was checked using the Basic Local Alignment Search Tool at NCBI (http://www.ncbi.nlm.nih.gov/BLAST). A list of the target genes and primer conditions is shown in Table 2.

$\boldsymbol{R T}-\boldsymbol{q P} \boldsymbol{C R}$. The primer concentrations used were 2 $\mu M$ for $G A D P H$ and HPRT1 and $5 \mu M$ for all other primers. During each run, a $5 \times$ dilution series was in- 
Table 1. Primers of the reference genes used for normalization of the reverse transcription-quantitative PCR data

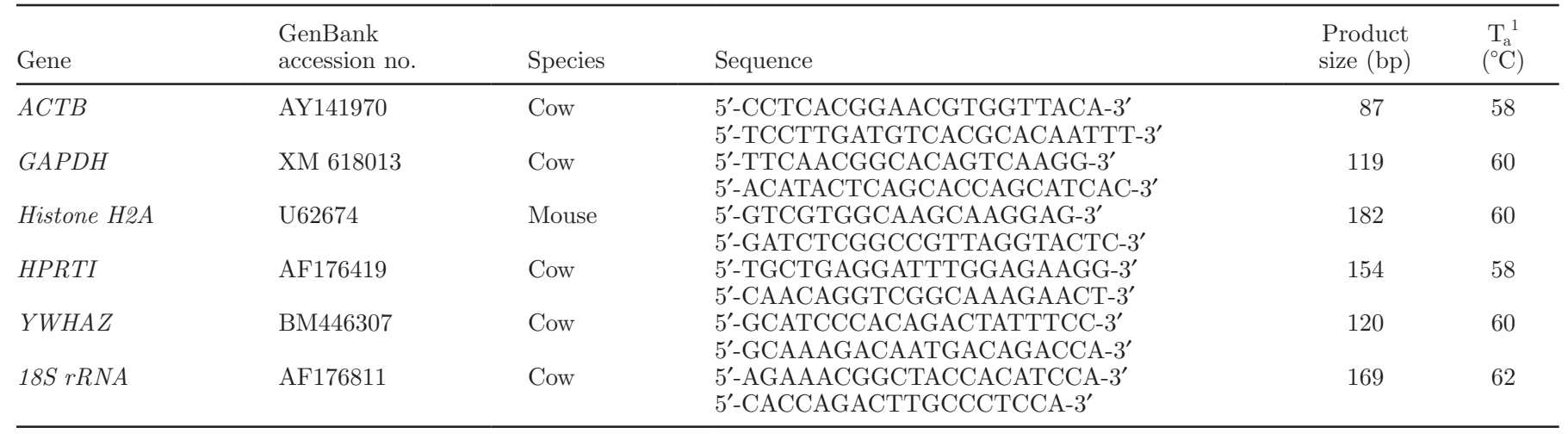

${ }^{1} \mathrm{~T}_{\mathrm{a}}=$ annealing temperature.

cluded to create a relative standard curve, which was used for PCR efficiency calculations. A no-template control was also added to every run. Absence of primer dimers was verified by melting curve analysis and by $2 \%$ gel agarose electrophoresis. The efficiencies were between 85.30 and $110.00 \%$, and primer dimers were absent in all reactions.

Gene Expression Measurement and Data Analysis. Cycle threshold values and raw expression data were determined for the reference genes and target genes on all samples $(10 \times$ diluted $)$. Next, obtained relative quantities were normalized by dividing the raw expression data of the target genes by the normalization factor, which is the geometric mean of the raw expression data of the 4 most stable reference genes. For all statistical analyses, these normalized expression data were converted to logarithmic values (Vandesompele et al. 2002). Data are presented as means \pm

Table 2. Primers of the toll-like receptor-4 (TLR4) receptor and associated genes for reverse transcription-quantitative PCR analysis

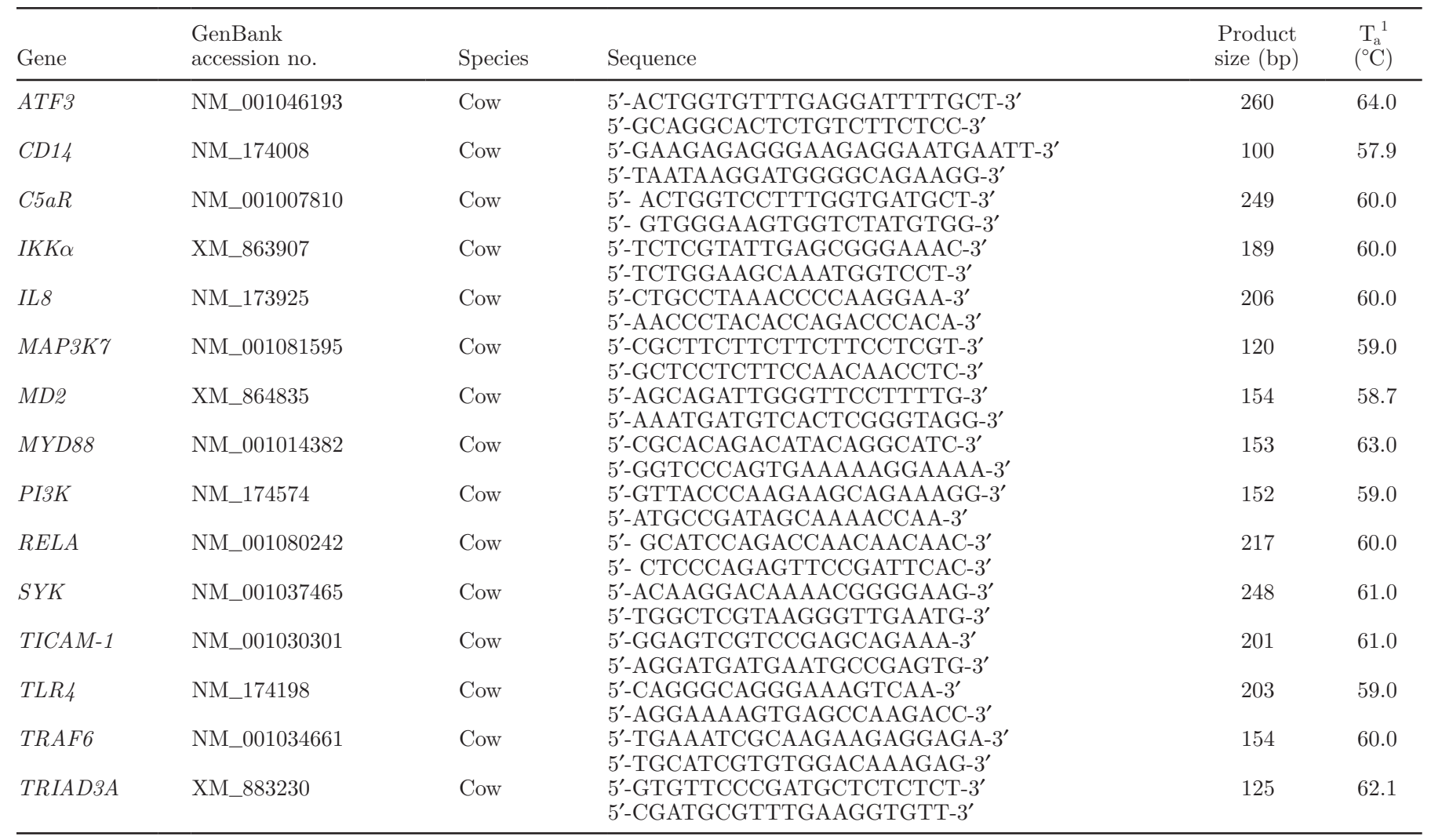

${ }^{1} \mathrm{~T}_{\mathrm{a}}=$ annealing temperature. 

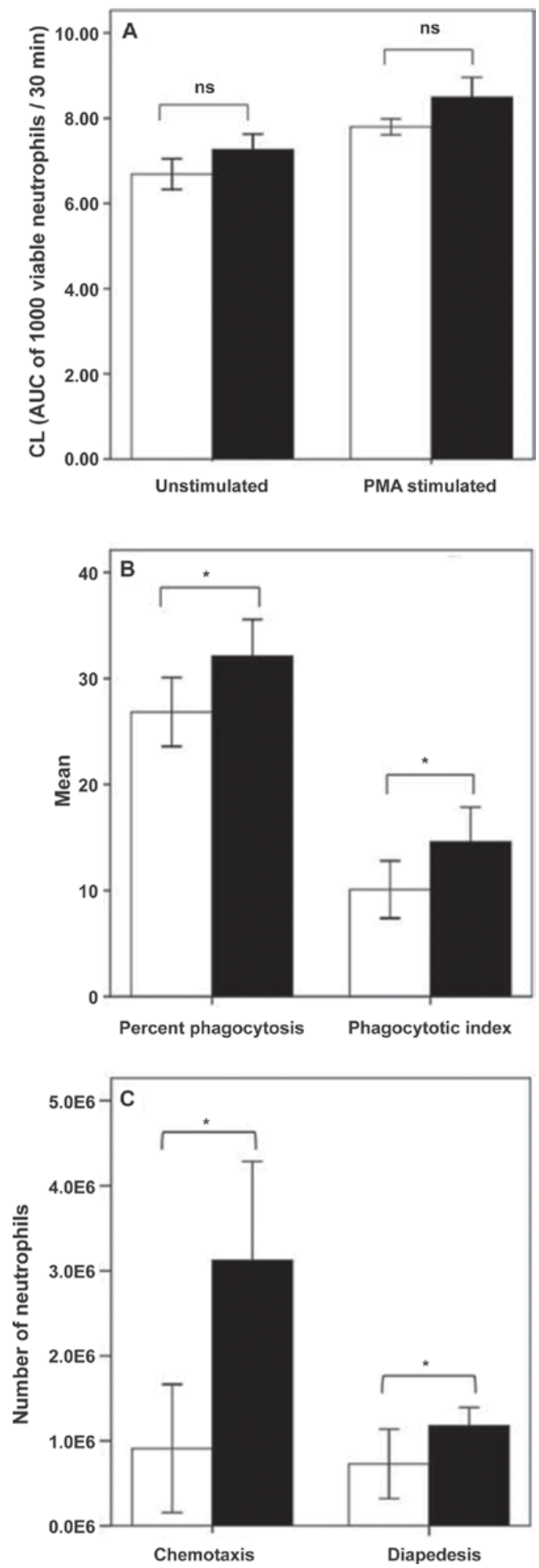

Figure 2. Neutrophil functions in early- (EL) and mid- (ML) lactating cows. Neutrophils were collected from 10 cows sampled $3 \mathrm{~d}$ postpartum ( $\square$ ) and 10 cows sampled 149 to $151 \mathrm{~d}$ postpartum (ם). A) Chemiluminescence (CL); B) mean percentage phagocytosis; and C) diapedesis and chemotaxis. AUC $=$ area under the curve. Asterisks denote a significant difference $(P<0.05)$ between the EL and ML cows.
SEM. To compare the 2 periods (EL and ML) with respect to different response variables [CTX, DIA, and unstimulated (REST)], a mixed model with lactation stage and their interactions as the fixed effects and cow as the random effect was used to study the relationship between stage of lactation and gene expressions before and after CTX and DIA. A fixed effect model with lactation stage as fixed effect was used to study the relationship between lactation stage and neutrophil function. Hypothesis testing was done at 5\% significance level. The normalized expression values $(\Delta \mathrm{Ct})$ of the target genes were compared (1) in each lactation stage between resting and migrated neutrophils and (2) between EL and ML by calculating $\Delta \Delta \mathrm{CT}$. The $\Delta \Delta \mathrm{CT}$ was used to calculate the fold change by performing a $2^{-\Delta \Delta \mathrm{CT}}$ calculation.

\section{RESULTS}

\section{Neutrophil Functions in EL Cows}

To quantify the expected impairment of neutrophil function in EL cows, the ability of isolated neutrophils to perform phagocytosis, oxidative burst, diapedesis, and chemotaxis was measured. Before this functional testing, a differential count of the isolated cells was performed and showed that $94.80 \% \pm 0.50$ of the isolated cells from EL cows and $94.20 \% \pm 0.70$ of the cells from ML cows had a neutrophilic phenotype. In the neutrophil population of EL cows, $86.90 \% \pm 1.20$ and in ML cows, $90.10 \% \pm 1.60$ were mature segmented neutrophils. All neutrophils had a viability of $>99 \%$.

Oxidative Burst. To measure the oxidative burst capacity of the neutrophils, the amount of reactive oxygen species (ROS) released by the neutrophils was calculated as the AUC. Unstimulated neutrophils from EL cows had a lower response compared with those of ML cows with an AUC of $6.86 \times 10^{6}$ and $8.48 \times$ $10^{6} \mathrm{RLU}$, respectively (Figure 2A). Stimulation of neutrophils with PMA increased the luminol-dependent response up to $8.27 \times 10^{7}$ for neutrophils from EL cows and $9.35 \times 10^{7}$ for neutrophils isolated from ML cows, which points toward a tendency for lower ROS production during EL, although this could not be confirmed statistically (Figure 2A).

Phagocytosis. The percentage of neutrophils performing phagocytosis of FITC-coated E. coli was $26 \%$ during EL and 32\% during ML (Figure 2B; $P<0.05$ ). In addition, neutrophils from EL cows contained, on average, $1.32 \pm 0.04$ bacteria, and those from ML cows contained $1.37 \pm 0.04$ bacteria (determined by phase contrast microscopy; $P>0.05$ ). The phagocytotic index was lower in EL cows compared with ML cows (9.49 \pm 1.30 vs. $14.60 \pm 1.30 ; P<0.05)$. 
DIA. Neutrophils added to the basal chamber of the DIA model started to transmigrate in response to C5a added at the apical chamber. After 90 min, $7.29 \times$ $10^{5}$ EL neutrophils were counted in the basal chamber, whereas $1.24 \times 106 \mathrm{ML}$ neutrophils had performed DIA at that time point (Figure $2 \mathrm{C} ; P<0.05$ ), indicatin that neutrophil recruitment was lower in EL cows.

$\boldsymbol{C T X}$. To quantify the efficiency of CTX, neutrophils were allowed to migrate through a porous membrane (12-mm diameter, $8.0-\mu \mathrm{m}$ pore size) in response to 50 $\mathrm{ng} / \mathrm{mL}$ C5a. After $90 \mathrm{~min}$, the neutrophils from ML cows were significantly more numerous in the lower chamber of the CTX model compared with the neutrophils from EL cows $\left(9.10 \times 10^{5}\right.$ vs. $3.12 \times 10^{6} ; P<$ 0.05 ), indicating that neutrophils from ML cows migrated faster under influence of a chemotactic gradient than did neutrophils from EL cows (Figure 2C).

Based on all these data, namely the fact that the neutrophil population isolated from the EL cows had a lower ability to perform phagocytosis, DIA, and CTX, these neutrophils were considered as immunecompromised neutrophils. In contrast, neutrophils from

Table 3. Effect of lactation stage and migration on the mRNA expression of the toll-like receptor-4 (TLR4) pathway and associated genes in bovine neutrophils

\begin{tabular}{|c|c|c|c|}
\hline Gene & $\begin{array}{l}\text { Neutrophil } \\
\text { group }^{1}\end{array}$ & $\begin{array}{l}\text { Early lactating } \\
\qquad(\mathrm{n}=10)\end{array}$ & $\begin{array}{l}\text { Mid lactating } \\
\quad(\mathrm{n}=10)\end{array}$ \\
\hline \multirow[t]{3}{*}{ ATF3 } & REST & $-1.062(0.200)$ & $-0.617(0.062)$ \\
\hline & DIA & $0.320^{\mathrm{a}}(0.071)$ & $0.043^{\mathrm{a}}(0.114)$ \\
\hline & CTX & $0.183^{\mathrm{a}}(0.112)$ & $0.281^{\mathrm{a}}(0.074)$ \\
\hline \multirow[t]{3}{*}{$C D 14$} & REST & $-0.044(0.093)$ & $-0.257(0.124)$ \\
\hline & DIA & $0.222(0.137)$ & $0.473^{\mathrm{a}}(0.129)$ \\
\hline & CTX & $0.233(0.138)$ & $0.233^{\mathrm{a}}(0.085)$ \\
\hline \multirow[t]{3}{*}{$C 5 a R$} & REST & $-0.190(0.075)$ & $0.085(0.042)$ \\
\hline & DIA & $0.068^{\mathrm{a}}(0.09)$ & $0.222(0.075)$ \\
\hline & CTX & $0.102^{\mathrm{a}}(0.100)$ & $0.219(0.114)$ \\
\hline \multirow[t]{3}{*}{$I K K \alpha$} & REST & $-0.014(0.079)$ & $0.054(0.042)$ \\
\hline & DIA & $-0.033(0.093)$ & $0.044(0.061)$ \\
\hline & CTX & $0.016(0.087)$ & $0.031(0.054)$ \\
\hline \multirow[t]{3}{*}{$I L 8$} & REST & $-2.085(0.214)$ & $-1.432(0.263)$ \\
\hline & DIA & $0.21^{\mathrm{a}}(0.152)$ & $0.213^{\mathrm{a}}(0.116)$ \\
\hline & CTX & $-0.267^{\mathrm{a}}(0.099)$ & $-0.096^{\mathrm{a}}(0.153)$ \\
\hline \multirow[t]{3}{*}{ МАР $3 K^{7}$} & REST & $-0.229(0.052)$ & $-0.071(0.078)$ \\
\hline & DIA & $-0.261(0.070)$ & $-0.340^{\mathrm{a}}(0.061)$ \\
\hline & CTX & $-0.232(0.083)$ & $-0.146(0.040)$ \\
\hline \multirow[t]{3}{*}{$M D^{2}$} & REST & $-0.104(0.091)$ & $0.003(0.046)$ \\
\hline & DIA & $-0.033(0.090)$ & $-0.085(0.031)$ \\
\hline & CTX & $-0.015(0.088)$ & $-0.146(0.045)$ \\
\hline \multirow[t]{3}{*}{ MyD88 } & REST & $0.092(0.088)$ & $0.201(0.034)$ \\
\hline & DIA & $0.081(0.095)$ & $0.128(0.045)$ \\
\hline & CTX & $0.178(0.085)$ & $0.117(0.039)$ \\
\hline \multirow[t]{3}{*}{ PIK3 } & REST & $0.061(0.096)$ & $0.144(0.029)$ \\
\hline & DIA & $-0.198^{\mathrm{a}}(0.130)$ & $-0.110^{\mathrm{a}}(0.075)$ \\
\hline & CTX & $-0.130(0.072)$ & $-0.168^{\mathrm{a}}(0.059)$ \\
\hline \multirow[t]{3}{*}{$R E L A$} & REST & $-0.521(0.087)$ & $-0.255(0.094)$ \\
\hline & DIA & $-0.541(0.088)$ & $-0.442(0.057)$ \\
\hline & CTX & $-0.404(0.123)$ & $-0.481(0.104)$ \\
\hline \multirow[t]{3}{*}{ Syk } & REST & $-0.263(0.067)$ & $-0.257(0.083)$ \\
\hline & DIA & $-0.242(0.096)$ & $-0.630^{\mathrm{a}}(0.086)$ \\
\hline & CTX & $-0.300(0.100)$ & $-0.436(0.057)$ \\
\hline \multirow[t]{3}{*}{ TICAM-1 } & REST & $-0.789(0.083)$ & $-0.631(0.086)$ \\
\hline & DIA & $-0.612(0.168)$ & $-0.533(0.163)$ \\
\hline & CTX & $-0.621(0.164)$ & $-0.785(0.128)$ \\
\hline \multirow{3}{*}{ TLR4 } & REST & $-0.422(0.084)$ & $-0.265(0.048)$ \\
\hline & DIA & $0.098^{\mathrm{a}}(0.071)$ & $0.175^{\mathrm{a}}(0.092)$ \\
\hline & CTX & $0.010^{\mathrm{a}}(0.094)$ & $0.220^{\mathrm{a}}(0.057)$ \\
\hline \multirow[t]{3}{*}{ TRAF6 } & REST & $-0.316(0.079)$ & $-0.061(0.038)$ \\
\hline & DIA & $-0.401(0.087)$ & $-0.206(0.043)$ \\
\hline & CTX & $-0.383(0.120)$ & $-0.217(0.077)$ \\
\hline \multirow[t]{3}{*}{ TRIAD3A } & REST & $-0.020(0.053)$ & $0.110(0.025)$ \\
\hline & DIA & $-0.015(0.073)$ & $-0.035(0.084)$ \\
\hline & CTX & $0.142(0.089)$ & $0.120(0.039)$ \\
\hline
\end{tabular}

${ }^{a}$ Significant difference in gene expression between REST and DIA or REST and CTX in each lactation stage. ${ }^{1} \mathrm{REST}=$ unstimulated neutrophils; DIA $=$ diapedesis; $\mathrm{CTX}=$ chemotaxis. 
Table 4. Fold decrease of differently expressed genes in neutrophils from cows in early lactation ${ }^{1}$

\begin{tabular}{lc}
\hline Gene & $\begin{array}{c}\text { Fold } \\
\text { decrease }\end{array}$ \\
\hline Unstimulated neutrophils & \\
ATF3 & 1.36 \\
C5aR & 1.21 \\
IL8 & 1.57 \\
RELA & 1.20 \\
TRAF6 & 1.19 \\
Diapedesis & \\
Syk & 1.31 \\
\hline
\end{tabular}

${ }^{1}$ Only those genes are shown for which the difference between earlyand mid-lactating cows was significant $(P<0.05)$. In the fold column, the downregulation of the gene is shown compared with the expression in neutrophils from ML cows. No significant differences in gene expression were found between early- and mid-lactating cows after chemotaxis.

the ML cows were fully competent and these immunecompetent cells were used as the normal control group for comparison. Next, the effect of lactation stage and DIA or CTX on the gene expression of selected members from the TLR4 pathway was analyzed.

\section{Effect of Lactation Stage on TLR4 and NF-KB Signaling in Unstimulated Neutrophils}

The mRNA transcription levels in unstimulated neutrophils were compared between cows in EL and ML. In unstimulated neutrophils, no effect for lactation stage was found for the genes of the receptor complex TLR $4 /$

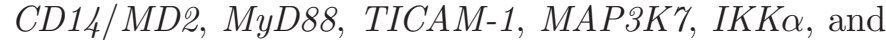
Syk (Tables 3 and 4). However, the mRNA transcription of TRAF6, RELA, and IL8 was decreased in neutrophils from EL cows. Expression of the endogenous inhibitors of the TLR4 pathway, TRIAD $3 A$ and PI3K, was not influenced by lactation stage, whereas expression of activating transcription factor gene, ATF3, was lower in EL cows compared with the ML cows (Tables 3 and 4$)$.

Chemotaxis through a porous membrane did not result in significant different mRNA expressions between cows in EL and ML (Tables 3 and 4). Lactation stage had no effect on the TLR4 pathway in the group of neutrophils that underwent DIA except for $S y k$, which was expressed at a lower level in ML cows (Tables 3 and 4$)$.

\section{Effect of Migration on TLR4 and NF-KB Signaling in Neutrophils}

Within each stage of lactation, the mRNA expression of the selected genes from unstimulated neutrophils was compared with the expression level after DIA or CTX.
In EL cows, significant upregulation of TLR4, ATF3 and IL8 was observed in neutrophils, after DIA as well as CTX (Tables 3 and 5; $P<0.05$ ). The TLR4 inhibitor PI3K was significantly downregulated $(P<$ 0.05) after DIA, whereas CTX did not influence its expression (Tables 3 and 5). No effect was seen of DIA or CTX on the expression of CD14, MD2, MyD88, TRAF6, TICAM-1, TRAF6, IKK, MAP3K 7, RELA, or TRIAD $3 A$ (Tables 3 and 5).

In ML cows, DIA and CTX induced a significant upregulation of TLR4, CD14, ATF3, and IL8 (Tables 3 and 5; $P<0.05$ ) and a downregulation of PI3K (Table 4; $P<0.05$ ). In contrast, mRNA levels of MAP3K 7 and Syk were significantly downregulated after DIA (Tables 3 and 5; $P<0.05$ ), whereas CTX did not alter the mRNA expression of these genes (Tables 3 and 5). No effect of DIA or CTX was seen on the mRNA expression of MDQ, MyD88, TICAM-1, TRAF6, IKK $\alpha, R E L A$, or TRIAD3A (Tables 3 and 5).

\section{Effect of Lactation Stage and Migration on C5aR}

As the bovine complement factor C5a was used as a chemoattractant for the DIA and CTX assays, the expression of the C5a receptor, C5aR, was analyzed in both lactation stages in unstimulated neutrophils and after DIA/CTX.

In unstimulated neutrophils, an effect of lactation stage was observed with significantly lower $C 5 a R$ expression in the immune-compromised neutrophils from EL cows (Figure 2; $P<0.05$ ) but not in ML neutrophils (Tables 3 and 4 ). Diapedesis or CTX increased the mRNA expression of $C 5 a R$ in EL cows $(P<0.05)$ but not in ML cows.

\section{DISCUSSION}

To the best of our knowledge, this is the first study evaluating the expression of genes of the TLR4 pathway

Table 5. Fold change of differently expressed genes in neutrophils from early-lactating (EL) and mid-lactating (ML) cows after migration ${ }^{1}$

\begin{tabular}{|c|c|c|c|c|}
\hline \multirow[b]{2}{*}{ Gene } & \multicolumn{2}{|c|}{ Chemotaxis } & \multicolumn{2}{|c|}{ Diapedesis } \\
\hline & EL & ML & EL & ML \\
\hline ATF3 & $0.42 \uparrow$ & $0.54 \uparrow$ & $0.38 \uparrow$ & $\overline{0.63 \uparrow}$ \\
\hline$C D 14$ & - & $0.60 \uparrow$ & - & $0.71 \uparrow$ \\
\hline C5aR & $0.82 \uparrow$ & - & $0.84 \uparrow$ & - \\
\hline IL 8 & $0.28 \uparrow$ & $0.40 \uparrow$ & $0.20 \uparrow$ & $0.32 \uparrow$ \\
\hline МАРЗК & - & - & - & $1.20 \uparrow$ \\
\hline PIK3 & - & $1.24 \downarrow$ & $1.20 \downarrow$ & $1.20 \downarrow$ \\
\hline Syk & - & - & - & $1.30 \downarrow$ \\
\hline TLR 4 & $0.74 \uparrow$ & $0.71 \uparrow$ & $0.70 \uparrow$ & $0.74 \uparrow$ \\
\hline
\end{tabular}

${ }^{1}$ Only those genes are shown for which the difference between resting and migrated neutrophils was significant $(P<0.05)$. $\downarrow$ indicates a downregulation and $\uparrow$ an upregulation of the gene expression compared with the expression in resting neutrophils before migration. 
in functionally characterized neutrophils between dairy cows during EL and ML. Messenger RNA originates from active genes and is used as a template for proteins that determine the neutrophilic phenotype. Although mRNA expression can be used to investigate intracellular events in the neutrophil, it should be mentioned that mRNA undergoes posttranscriptional modifications; thus, measuring mRNA alone has certain limitations when trying to understand innate immunity. Still, it was our specific goal to initiate a study to investigate the expression of genes of the TLR4 pathway in immunecompromised neutrophils, based on the hypothesis that this pathway could be involved in postpartum E. coli mastitis (De Schepper et al., 2008). Recent studies suggest an important role of TLR4 in endometritis and mastitis, which are common postpartum diseases in dairy cow. Herath and coworkers (2009) observed an upregulation of TLR4 in the endometrium of infertile animals with persistent endometritis compared with fertile, healthy postpartum cows. They hypothesize that an unlimited innate response could be at the basis of endometritis development. A similar result was found by Petzl et al. (2008) in mammary gland tissue from infected udder quarters in an E. coli-induced mastitis model. Interestingly, a relationship between impaired functions of peripheral blood neutrophils during the periparturient period and metritis (Hammon et al., 2006) on the one hand and mastitis (Heyneman et al., 1990) on the other hand has been found. Nothing is known yet about the expression of the TLR4 pathway in immune-compromised neutrophils. Therefore, this preliminary study was set up to compare the expression of TLR4-related genes in immune-compromised and immune-competent neutrophils. First, in vitro leukocyte function tests were performed to characterize the neutrophils. In agreement with previous reports (Burvenich et al., 2003), we observed that blood neutrophils isolated from cows in EL showed a decreased CTX, DIA, and phagocytosis compared with neutrophils isolated from cows in ML. Oxidative burst, measured by ROS production after PMA stimulation, had a tendency to be lower in neutrophils from EL cows but this could not be statistically confirmed. Failure to identify this statistical difference might be due to the individual variation of the cows, the relatively small sample size, or the method. The generation of ROS is mediated by a membrane-associated complex referred to as NADPH oxidase (Babior, 1999). This enzyme complex can be activated by zymosan and protein kinase $\mathrm{C}$ activators such as PMA. Its activation leads to the production of intracellular and extracellular ROS. Rinaldi et al. (2007) analyzed different assays for the measurement of ROS in bovine neutrophils. Those authors concluded that luminol-dependent chemiluminescence reflected in- tracellular increases in ROS production or extracellular increases in ROS other than superoxide and hydrogen peroxide. This might explain why we could not detect a significant decrease in respiratory burst in EL cows.

Based on the functional profiles, neutrophils from EL cows were considered immune-compromised and those from ML cows were considered immune-competent and were used as a control group. Next, we wanted to know if this immune-compromised neutrophil status was accompanied by alterations in the mRNA expression of TLR4-related genes.

In unstimulated neutrophils, mRNA expression of TLR4-associated genes was virtually indistinguishable between EL and ML cows, with the exception of a decreased expression of TRAF6, RELA, ATF3, and IL8 in neutrophils from EL cows. The TRAF6 gene acts downstream in the TLR4 pathway and is a key gene in the activation of $N F-\kappa B$, which eventually leads to the production of inflammatory cytokines (Inoue et al., 2007). We also observed a significant decrease in the transcription of the cytokine $I L 8$, tempting us to hypothesize that the decreased TRAF6 expression was translated in a decreased $I L 8$ expression. In a microarray study comparing the expression levels of several genes in blood neutrophils at parturition and $7 \mathrm{~d}$ before parturition, a similar downregulation of TRAF6 expression was reported but was not accompanied by decreased IL8 expression (Madsen et al., 2004). This discrepancy could be due to the fact that in the present study, neutrophils were used from cows $3 \mathrm{~d}$ postparturition and 149 to $151 \mathrm{~d}$ postparturition. It could also be explained by the fact that not all genes expressed by neutrophils were analyzed in our study. For example, it has been shown that TLR4 signaling is controlled by intracellular regulators such as PI3K, ATF3, and TRIAD3A, but membrane-associated receptors such a ST2 (a toll-IL-1R domain receptor) and TRAILR (a tumor necrosis factor receptor) have also been described to regulate TLR4 signaling (Liew et al., 2005). This suggests that complex regulatory networks exist and that the downregulation of $I L 8$ could be caused by regulatory genes not included in the present study.

Next, we wanted to know if migration, consisting of DIA and CTX, affects TLR 4 signaling in neutrophils. The mammary epithelial model system used in this study has been successfully used in our laboratory to study neutrophil DIA in vitro. It consists of a monolayer of BME-UV cells seeded on a collagen-coated membrane insert and uses C5a as a chemoattractant (Smits et al., 1996). For CTX, a similar model was used without the epithelial monolayer and collagen coating. Because C5a was used for the migration experiments, one could argue that the chemoattractant by itself was responsible for the observed changes in mRNA expres- 
sions. However, in a previous study at our laboratory, the direct effect of C5a on the gene expression of the TLR4 pathway in neutrophils was determined and it was found that gene expressions were not influenced by the lactation stage $[$ M. G. H. Stevens, L. J. Peelman. B. De Spiegeleer, A. Pezeshki, G. R. Van De Walle, L. Duchateau, C. Burvenich, and R. Rainard (Laboratoire de Pathologie Infectieuse et Immunologie, Institut National de la Recherche Agronomique, Nouzilly, France), unpublished data]. Therefore, we conclude that the observed differences in gene expression after migration seen in the present study between neutrophils from EL and ML cows are not caused by the chemoattractant but are the result of neutrophil migration.

The salient findings were that both DIA and CTX induced the mRNA transcription of TLR4,IL8, and ATF3 in neutrophils from EL and ML cows. The primary function of the TLR4 receptor is to recognize PAMP from invading pathogens (Beutler et al., 2001). Downstream signaling events will then ultimately result in the production of proinflammatory cytokines such as IL-8. To our knowledge, nothing is known yet about the effect of DIA or CTX on TLR 4 in neutrophils, but our data suggest that neutrophil migration is able to regulate the expression of the TLR4 receptor to prepare the neutrophils for the recognition of pathogens and triggers the transcription of $I L 8$.

TLR4's co-receptor CD14 was significantly upregulated in immune-competent neutrophils after DIA and CTX, but no upregulation was seen in the immunecompromised neutrophils. No explanation for this unexpected observation of a TLR 4 upregulation in the absence of $C D 14$ upregulation in immune-compromised neutrophils can be given, but it might suggest that these neutrophils have dysfunctional LPS sensing after DIA, which has been hypothesized by De Schepper et al. (2008). However, it should be taken into account that upregulation at the mRNA level does not necessary translate into a functional effect and therefore these observations should be confirmed at protein level.

The TLR4 pathway is strictly regulated, and intracellular and transmembrane proteins have been identified that negatively regulate TLR4 signaling (Liew et al., 2005). For example, ATF3 functions as a negative regulator of the TLR4 receptor by inhibiting NF-kB (Gilchrist et al., 2006). Here, we observed an increased level of ATF3 mRNA in neutrophils after DIA, which may suggest a protective feedback on the NF- $\mathrm{BB}$ signaling pathway. A tendency for decreased expression of $R E L A$ was noted but further research is needed to elucidate this.

Although migration induced different changes in mRNA expression in neutrophils from EL and ML cows, mRNA expression after migration of most of the
$T L R$ 4-related genes was not significantly different except for that of Syk. We found that DIA but not CTX induced significant downregulation of $S y k$ in immunecompetent neutrophils, whereas DIA did not change Syk expression in the immune-compromised neutrophils. No explanation has been found to date for the unchanged Syk expression after DIA in immune-compromised neutrophils, which was in contrast to findings in the immune-competent neutrophils. However, Syk could be an attractive candidate for further research because it has been reported that type I hypersensitivity reactions such as allergic rhinitis and systemic anaphylaxis seem to partially depend on Syk activation, and targeting Syk has therefore been proposed as a means for treatment (Wong et al., 2004).

\section{CONCLUSIONS}

We performed a genomic scan by qPCR analysis of the TLR4 pathway in neutrophils from EL and ML cows. To our best knowledge this is the first study to show that neutrophil migration (DIA and CTX) changes mRNA expression in bovine neutrophils. Moreover, migration differently influenced the mRNA expression of TLR 4related genes in EL and ML cows. This study shows that the perturbations in neutrophil functions during EL are accompanied by modulation of TLR4-related genes. These data can contribute to the understanding of the mechanisms to explain the relationship between stage of lactation and risk of severe E. coli mastitis.

\section{ACKNOWLEDGMENTS}

This study was financed by the Fonds Wetenschappelijk Onderzoek (FWO) Vlaanderen (Brussels, Belgium; grant \# G.0050.06N). The authors greatly acknowledge Pascal Rainard (INRA UR1282 Laboratoire d'Infectiologie Animale et Santé Publique, Nouzilly, France) for providing the purified bovine C5a. We also thank M. Van Poucke (Ghent University) for support and advice and Carolien Rogiers (Ghent University) for her technical assistance.

\section{REFERENCES}

Akira, S., and K. Hoshino. 2003. Myeloid differentiation factor 88-dependent and independent pathways in toll-like receptor signaling. J. Infect. Dis. 187(Suppl 2):S356-S363.

Arndt, P. G., N. Suzuki, N. J. Avdi, K. C. Malcolm, and G. S. Worthen. 2004. Lipopolysaccharide-induced c-Jun NH2-terminal kinase activation in human neutrophils: Role of phosphatidylinositol 3-kinase and Syk-mediated pathways. J. Biol. Chem. 279:10883-10891.

Babior, B. M. 1999. NADPH oxidase: An update. Blood 93:14641476.

Beutler, B., X. Du, and A. Poltorak. 2001. Identification of toll-like receptor 4 (Tlr4) as the sole conduit for LPS signal transduction: Genetic and evolutionary studies. J. Endotoxin Res. 7:277-280. 
Burvenich, C., M. J. Paape, A. W. Hill, A. J. Guidry, R. H. Miller, R. Heyneman, W. D. Kremer, and A. Brand. 1994. Role of the neutrophil leucocyte in the local and systemic reactions during experimentally induced $E$. coli mastitis in cows immediately after calving. Vet. Q. 16:45-50.

Burvenich, C., V. Van Merris, J. Mehrzad, A. Diez-Fraile, and L. Duchateau. 2003. Severity of E. coli mastitis is mainly determined by cow factors. Vet. Res. 34:521-564.

Chaudhary, A., T. M. Fresquez, and M. J. Naranjo. 2007. Tyrosine kinase Syk associates with toll-like receptor 4 and regulates signaling in human monocytic cells. Immunol. Cell Biol. 85:249-256.

Chuang, T. H., and R. J. Ulevitch. 2004. Triad3A, an E3 ubiquitinprotein ligase regulating toll-like receptors. Nat. Immunol. 5:495502.

De Ketelaere, A., K. Goossens, L. Peelman, and C. Burvenich. 2006. Validation of internal control genes for gene expression analysis in bovine polymorphonuclear leucocytes. J. Dairy Sci. 89:40664069.

De Schepper, S., A. De Ketelaere, D.D. Bannerman, M.J. Paape, L. Peelman, and C. Burvenich. 2008. The toll-like receptor-4 (TLR4) pathway and its possible role in the pathogenesis of Escherichia coli mastitis in dairy cattle. Vet. Res. 39:05.

Fitzgerald, K. A., D. C. Rowe, and D. T. Golenbock. 2004. Endotoxin recognition and signal transduction by the TLR4/MD2-complex. Microbes Infect. 6:1361-1367.

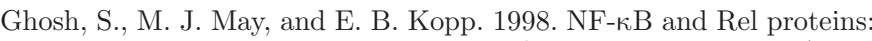
Evolutionarily conserved mediators of immune responses. Annu. Rev. Immunol. 16:225-260.

Gilchrist, M., V. Thorsson, B. Li, A. G. Rust, M. Korb, J. C. Roach, K. Kennedy, T. Hai, H. Bolouri, and A. Aderem. 2006. Systems biology approaches identify ATF3 as a negative regulator of tolllike receptor 4 . Nature 441:173-178.

Hammon, D. S., I. M. Evjen, T. R. Dhiman, J. P. Goff, and J. L. Walters. 2006. Neutrophil function and energy status in Holstein cows with uterine health disorders. Vet. Immunol. Immunopathol. 113:21-29.

Herath, S., S. T. Lilly, N. R. Santos, R. O. Gilbert, L. Goetze, C. E. Bryant, J. O. White, J. Cronin, and I. M. Sheldon. 2009. Expression of genes associated with immunity in the endometrium of cattle with disparate postpartum uterine disease and fertility. Reprod. Biol. Endocrinol. 7:55.

Heyneman, R., C. Burvenich, and R. Vercauteren. 1990. Interaction between the respiratory burst activity of neutrophil leucocytes and experimentally induced Escherichia coli mastitis in cows. J. Dairy Sci. 73:985-994.

Hoeben, D., E. Monfardini, G. Opsomer, C. Burvenich, H. Dosogne, A. De Kruif, and J. F. Beckers. 2000. Chemiluminescence of bovine polymorphonuclear leucocytes during the periparturient period and relation with metabolic markers and bovine pregnancyassociated glycoprotein. J. Dairy Res. 67:249-259.

Inoue, J., J. Gohda, and T. Akiyama. 2007. Characteristics and biological functions of TRAF6. Adv. Exp. Med. Biol. 597:72-79.
Laird, M. H., S. H. Rhee, D. J. Perkins, A. E. Medvedev, W. Piao, M. J. Fenton, and S. N. Vogel. 2009. TLR4/MyD88/PI3K interactions regulate TLR4 signaling. J. Leukoc. Biol. 85:966-977.

Liew, F. Y., D. Xu, E. K. Brint, and L. A. O'Neill. 2005. Negative regulation of toll-like receptor-mediated immune responses. Nat. Rev. Immunol. 5:446-458.

Madsen, S. A., L. C. Chang, M. C. Hickey, G. J. Rosa, P. M. Coussens, and J. L. Burton. 2004. Microarray analysis of gene expression in blood neutrophils of parturient cows. Physiol. Genomics $16: 212-221$

Mehrzad, J., H. Dosogne, F. Vangroenweghe, and C. Burvenich. 2001. A comparative study of bovine blood and milk neutrophil functions with luminol-dependent chemiluminescence. Luminescence $16: 343-356$

Mócsai, A., J. Ruland, and V. L. Tybulewicz. 2010. The SYK tyrosine kinase: A crucial player in diverse biological functions. Nat. Rev. Immunol. 10:387-402.

Petzl, W., H. Zerbe, J. Günther, W. Yang, H. M. Seyfert, G. Nürnberg, and H. J. Schuberth. 2008. Escherichia coli, but not Staphylococcus aureus triggers an early increased expression of factors contributing to the innate immune defense in the udder of the cow. Vet. Res. 39:18.

Rinaldi, M., P. Moroni, M. J. Paape, and D. D. Bannerman. 2007. Evaluation of assays for the measurement of bovine neutrophil reactive oxygen species. Vet. Immunol. Immunopathol. 115:107125.

Sada, K., T. Takano, S. Yanagi, and H. Yamamura. 2001. Structure and function of Syk protein-tyrosine kinase. J. Biochem. 130:177186.

Sears, P. M., M. J. Paape, R. E. Pearson, and F. C. Gwazdauskas. 1978. Comparison between tail vein and jugular vein cannulation in cattle. J. Dairy Sci. 61:974-979.

Siemens, D. W., I. A. Schepetkin, L. N. Kirpotina, B. Lei, and M. T. Quinn. 2007. Neutrophil isolation from nonhuman species. Methods Mol. Biol. 138:21-34.

Smits, E., E. Cifrian, A. J. Guidry, P. Rainard, C. Burvenich, and M. J. Paape. 1996. Cell culture system for studying bovine neutrophil diapedesis. J. Dairy Sci. 79:1353-1360.

Vandesompele, J., K. De Preter, F. Pattyn, B. Poppe, N. Van Roy, A. De Paepe, and F. Speleman. 2002. Accurate normalization of realtime quantitative RT-PCR data by geometric averaging of multiple internal control genes. Genome Biol. 3:RESEARCH0034.

Vangroenweghe, F., I. Lamote, and C. Burvenich. 2005. Physiology of the periparturient period and its relation to severity of clinical mastitis. Domest. Anim. Endocrinol. 29:283-293.

Wong, B. R., E. B. Grossbard, D. G. Payan, and E. S. Masuda. 2004. Targeting Syk as a treatment for allergic and autoimmune disorders. Expert Opin. Investig. Drugs 13:743-762. 